\section{Cardiomyopathy and Myocardial Biopsy}

Edited by M. Kaltenbach, F. Loogen \& E. G. J. Olsen. Pp. 337, illustrated. Berlin, Heidelberg and New York: Springer-Verlag, 1978. Price not given.

The aim of this book is to give an up to date account of recent developments in the field of cardiomyopathy. This is particularly orientated to the use of endomyocardial biopsy in the diagnosis and prognosis of patients with cardiomyopathy. Many of the authors come from Germany, although there are a few outside contributors, either from America or England.

The greatest emphasis in this book is on the use of myocardial biopsy and the analysis of small pieces of myocardial tissue thus obtained in the diagnosis and prognosis of the cardiomyopathies. Correspondingly, it is of particular interest to the morbid anatomist, and a large number of light microscopy and electron microscopy figures are illustrated. Most chapters take the format of a standard scientific paper with a reference section at the end. There are some chapters devoted to experimental cardiomyopathies. The hypertrophic and congestive cardiomyopathies are particularly emphasized in this book and little emphasis is made on any of the other forms of cardiomyopathy, e.g. restrictive, constrictive, hypertrophic and non-obstructive.

This book has a limited scope for readership, being particularly beneficial to the cardiologist interested in cardiomyopathy or the cardiac pathologist. It suffers from a slightly parochial view in being mainly German in authorship and would have had a wider field of application if contributions had been obtained from a more international pool. It provides a good update to the clinician particularly interested in this problem, although it has to be stated that there are no sections dealing with sub-cellular enzyme analysis or myocardial substrate analysis which would appear to be the most worthwhile fields for analysis of myocardial biopsy specimens.

This book has a limited scope owing to its specialist nature but is easily readable and provides a good update to either the specialist or the clinician wishing to know more of the myocardial biopsy technique and the cardiomyopathies.

\section{A Colour Atlas of Cardiac Pathology}

By GeOFFrey FARRER-Brown. Pp. 159, illustrated. London: Wolfe Medical Publications Ltd, 1977. Hard cover $£ 13.00$.

In his preface the author states that the Atlas is intended as a visual aid to a mixed readership ranging from senior clinical students to junior pathologists as well as other interested hospital staff. This wide aim plus the financial constraints of a wholly colour-illustrated book indicates that the intent is mainly to whet the appetite. This is not a criticism because to create interest is the foundation of teaching. Indeed the Atlas will surely succeed in its aims. The visual appeal is immediate and the whole book can be looked at and the legends read in an hour or two.

First there is instruction in how to remove and examine the heart at post-mortem followed by a description of the normal adult heart in its gross, microscopic and ultra-microscopic features.

Some congenital malformations are then illustrated, the material being derived as elsewhere in the book both from fresh material and from museum specimens. These malformations are notoriously hard to depict clearly especially when only one or, at most, two views of a specimen are included. An understanding of the bare essentials of the anatomy of the tetralogy of Fallot is hardly furthered by the picture of the infant heart and the single view of a double outlet right ventricle merely fills the space adjacent to the text. It is a pity too to include the fanciful or unproved in the short descriptive legends; it has been suggested that left sided heart disease with features similar to hypertrophic cardiomyopathy may be associated with congenital pulmonary valve stenosis in some patients but such cases are certainly exceptional amongst cases of this common and simple congenital valvar malformation.

The sections on the pericardium, ventricular hypertrophy, primary cardiomyopathies and rheumatic heart disease are very adequate in depicting the important disorders and for the most part the quality of the illustrations is outstanding. The chapters on the blood supply of the heart, coronary artery atheroma, myocardial infarction and its complications and the myocardial vasculature following myocardial infarction are outstanding reflecting as they do the author's important contributions to our knowledge of the subject. In view of the importance of the subject the imbalance given to the book in the amount of detail permitted in these chapters is well justified by the size of the problem and the personal expertise of the author.

The commonest valve disease of all, calcific aortic stenosis, seems to be rather thrown away under the heading of 'other valve conditions' but the illustrations and information are there just the same. The floppy mitral valve finds itself in the same area alongside carcinoid heart disease and Lambl's excrescences. The final chapters are related to infective endocarditis and myocarditis followed by familial diseases, the conduction system of the heart and cardiac tumours.

The author is to be congratulated on producing a lovely atlas, beautiful to look at, interesting, informative and not even outrageously expensive. I hope it will have the success it deserves.

\section{A Computer Model of Human Respiration}

By C. J. Dickinson. Pp. 256, illustrated. Lancaster: MTE Press, 1977. Hardback $£ 14.95$.

This book describes a computer programme by means of $\infty$ which all the measurable effects of variations in any of the many factors which affect respiration may be predicted; in the words of the author it presents a model 'as a co-operative human subject who will permit any, even lethal experiments to be performed upon him'. Its outstanding achievement is that it is one of the first attempts to provide a thoroughly integrated view of respiratory physiology based upon the enormous body of experimental data that has been accumulated during the past 80 years or so. In my opinion, it is comparable with Sherrington's book on the nervous system in that it collates all the important isolated discoveries and concepts that have appeared to date and welds them into a comprehensible whole. The fact that this remarkable achievement has been linked to the construction of a computer programme in no way detracts from the clarity of presentation; indeed, it emphasizes it. In case any would-be reader should fear that a knowledge of mathematics, computer techniques and programming is a necessary prerequisite to the profitable study of the book, the author himself states that he is no mathematician and that his approach to the mathematical problems posed has been intuitive rather than formal and exact and that he has used the computer as a tool which can substitute for mathematical skill in certain circumstances; we would not all agree with his further remark that it can also replace native intelligence! Nevertheless, any reader will find that the book must be read with the closest attention - there are not many sentences that can be missed without losing the thread of the arguments and descriptions. In general, the writing is clear and precise and avoids (thank goodness) the obscurities of computer jargon. It is sometimes difficult to remember when reading, the meanings of such $C$ symbols as XDSPA which stands for 'extra dead space specified by altered function tests for specified subjects'. This $\stackrel{\mathscr{S}}{+}$ kind of symbolism is an unavoidable adjunct to programming; after reading a few pages of the book the logic behind 